\title{
Langmuir films stability phenomenon of glycerol dialkyl nonitol tetraether at the air-water interface for variations spreading time
}

\author{
Sri Vidawati ${ }^{*}$, Udo Bakowsky ${ }^{2}$, Ulrich Rothe ${ }^{3}$ \\ ${ }^{1}$ Department of Physics, Faculty of Natural Science and Mathematics, University Indonesia, Depok, Indonesia \\ ${ }^{2}$ Department of Pharmaceutical Technology, Philipps University Marburg, Marburg, Germany \\ ${ }^{3}$ Institute of Physiological Chemistry, Faculty of Medicine, University Halle-Wittenberg, Halle/Saale, Germany \\ Email: ${ }^{*}$ sri.vidawati@ui.ac.id
}

Received 11 May 2012; revised 21 June 2012; accepted 29 June 2012

\begin{abstract}
Langmuir films behavior of asymmetric glycerol dialkyl nonitol tetraether (GDNT) lipids at the air-water interface (temperature $19^{\circ} \mathrm{C} \pm 1^{\circ} \mathrm{C}$ ) investigated the stability of the mean ratio of Langmuir films for different spreading time prior to the transfer film. Floating monolayer films stability is an important parameter to obtain high-quality Langmuir-Blodgett films for their aplications. Hysteresis loop of the compression-decompression $(\mu-\mathrm{A})$ isotherms of Langmuir film GDNT used to analyze the thermodynamic stability of the monolayer films and its features. Langmuir films stability of tetraether lipid GDNT shown more stable at the time of spreading longer than a short time.
\end{abstract}

Keywords: Asymmetric Glycerol Dialkyl Nonitol Tetraether Lipid; Surface Pressure-Area Isotherm; Langmuir Films Stability; Hysteresis Loop

\section{INTRODUCTION}

Amphiphiles Langmuir films on water have been intensively studied as model systems for two-dimensional matter [1], the cell membrane [2], and more recently for use in nanoscience and nanotechnology. Langmuir films, which are formed when a solution of non-soluble amphiphilic molecules spreads out by means of a solvent on the water's surface, are of great interest in biology as model membranes [3], in molecular electronics and specialized in the manufacture of electrodes [4] and biosensors [5].

Monolayers at the air-water interface provide an important and convenient model system to investigate the experimental chemical and biological problems. There are many other reasons to study the Langmuir monolayer films. One of the aspects related to the elaboration of nanotechnology growth controlled nanoscale systems. Gen-

${ }^{*}$ Corresponding author. erally speaking, nanobiotechnology requires the organization of atoms and molecules into a two- or three-dimensional space. The efficiency of nanofabrication strategies and recent developments of methods enabling the direct characterization at the molecular scale open up new routes in the development of self-organized nanostructures. Unique geometry of the air-water interface has allowed the measurement of certain macroscopic properties of these systems (eg, pressure-area ( $\mu$-a) isotherms), the experimental difficulties inherent in collecting data interface has limited spectroscopic studies. Analysis of surface structures and features of the interface is very important to compare and understand the properties of nanosystems. Analysis at the molecular and supramolecular biological systems, such as cell membranes, is an outstanding model to develop "smart nanostructures" based on molecular self-assembly of biological macromolecules. The potential of two-dimensional molecular self-assemblies is clearly illustrated by Langmuir films of lipid molecules.

Archaebacteria, which proliferate under extreme environments, are one class of organisms that have been found to provide a rich source of inspiration. Archaebacterial tetraether lipids very different from the conventional lipids in the structure and physicochemical properties. They are based on ether linkages instead of ester linkages and contain biphytanyl chains instead of fatty acyl chains. Membranes of Sulfolobus acidocaldarius mainly contain two classes of tetraethers, the glycerol dialkyl glycerol tetraethers (GDGTs) and the glycerol dialkyl nonitol tetraethers (GDNTs). Both lipid species containning two nonequivalent polar heads linked by two hydrophobic C40 phytanyl chains, with up to four cyclopentane rings per chain. GDGT contains two glycerol moieties, while in GDNT one of the glycerol moieties is substituted by nonitol, a polyol with 9 carbon atoms

Sulfolobus acidocaldarius can grow at temperatures up to $85^{\circ} \mathrm{C}$ and $\mathrm{pH} 2-3$. Archaebacterial membrane adapted such extreme environmental conditions are considered 
very stable. Tetraether lipid membranes formed are more resistant to oxidation and high temperature because of their ether linkage than the more labile ester links found in lipids from Bacteria and Eukaryota [6].

Monolayer films formed from archaeal tetraether lipids at the air-water interface, it is not easy to distinguish between two possible molecular configurations, and construction of the model clearly has been difficult $[7,8]$. The relatively stiff tetraether lipids symmetric as well asymmetric can adopt several conformative supramolecular arrangements. Driving force in all cases is the hydrophobic interaction between the tetraether core thus inducing domain formation already very early after spreading of the lipids at the water surface. At this point the molecules lie flat on the subphase forming float-like domains which later after compressing begin to elevate and adopt a horseshoe conformation which at the end flip up into an upright conformation [9]. Stability of tetraether lipids monolayer films on solid substrates also depends on the properties of the substrates surface [10].

According Bakowsky et al. (2000) [11], the organization tetraether lipids Langmuir films at the air-water interface strongly depends on the spreading time and lateral surface pressure. Floating monolayer stability is a crucial parameter to obtain high-quality Langmuir-Blodgett films. In this paper, we report the comparative stability of Langmuir films of tetraether lipid GDNT at the air water interface with different spreading time.

\section{MATERIALS AND METHODS}

\subsection{Lipids and Other Materials}

Lipids from the archaebacteria Sulfolobus acidocaldarius were used for the experiments. The lipids were prepared from the dried bacteria according to the method by du Plesis (2008) [12]. The lipid was lyophilised at $10^{-2}$ Torr and stored over phosphopentoxide. All other chemicals were obtained from Merck (Damstadt) or Sigma (Deisenhofen). The Langmuir film experiments were performed with tetraether lipids of the highest available purity. The chemical structure of GDNT is shown in Figure 1.

\subsection{Langmuir Film Experiments}

The original Langmuir method was based on directly measuring the outwards horizontal force exerted on a floating

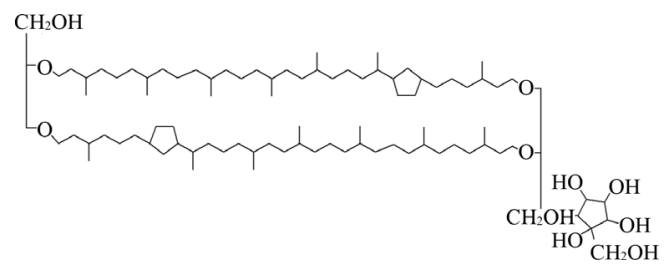

Figure 1. Chemical structure of GDNT (glycerol dialkyl nonitol tetraether). barrier that divides the film-covered surface from a clean surface. The spread monolayer can be compressed by means of a movable barrier while the surface pressure and the area are continuously recorded. Surface potential-area isotherms are also recorded.

All surface pressure-area isotherms were measured using a computerized Langmuir trough, manufactured by a commercial film balance company ( $\mathrm{R} \& \mathrm{~K} \mathrm{GmbH}$, Mainz, Germany), with a rectangular thermostated Teflon trough (area $418 \mathrm{~cm}^{2}, 1 \mathrm{~cm}$ depth). For the film balance experiments the tetraether lipids were dissolved in chloroform:methanol at 2:1 (v:v) consisting of a concentration $1.0 \mathrm{mmol} / \mathrm{l}$. Pure water of Milli Q quality (18.2 M $\Omega, \mathrm{pH}$ 5.6) was used as a subphase. All experiments were performed at a temperature of $19^{\circ} \mathrm{C} \pm 1^{\circ} \mathrm{C}$. After spreading, the lipid films were equilibrated at zero surface pressure before the measurements were taken. For each isotherm experiment, $30 \mu \mathrm{l}$ of $1.0 \mathrm{mmol} / \mathrm{l}$ for the GDNT solution in chloroform was spread onto the subphase. The compression-decompression was started $1 \mathrm{~h}$ and $15 \mathrm{~h}$ after spreading. All films were compressed-decompressed at a constant speed of $0.045 \mathrm{~cm}^{2} \cdot \mathrm{s}^{-1}\left(2.5 \times 10^{-2} \mathrm{~nm}^{2} \cdot \mathrm{s}^{-1}\right.$ per molecule).

\section{RESULT}

Recorded pressure vs. area/molecule (л/A) isotherms for the compression-decompression Tetraether lipids Langmuir films GDNT cycles with different time spread is shown in Figures 2(a) and (b).

For each compression-decompression cycle the samples compressed to a surface pressure approaching the collapse pressure and then decompressed to zero surface pressure. The first compression-decompression cycle is attributed to a difference in the organization and disorganization of the molecules or to the domains formed during compression reverting to their original state after decompression. The second compression-decompression began after both lipid films have been equilibrated at zero surface pressure for 1 hour, and then the third cycle begins after the second cycle has been equilibrated to zero surface pressure for 1 hour.

Compression-decompression hysteresis cycles performed, and resulting compression-decompression curves showing the thermodynamic stability of the tetraether lipids GDNT monolayers [10].

\section{DISCUSSION}

Phase behavior and molecular organization tetraetherlipids are of special interest, because the lipids of very stable with potential pharmaceutical applications. However, the findings are contradictory and many opinions remain.

Many studies on Langmuir film GDNT determined. The molecules studied are shown Rolandi et al. (1986) [7] 


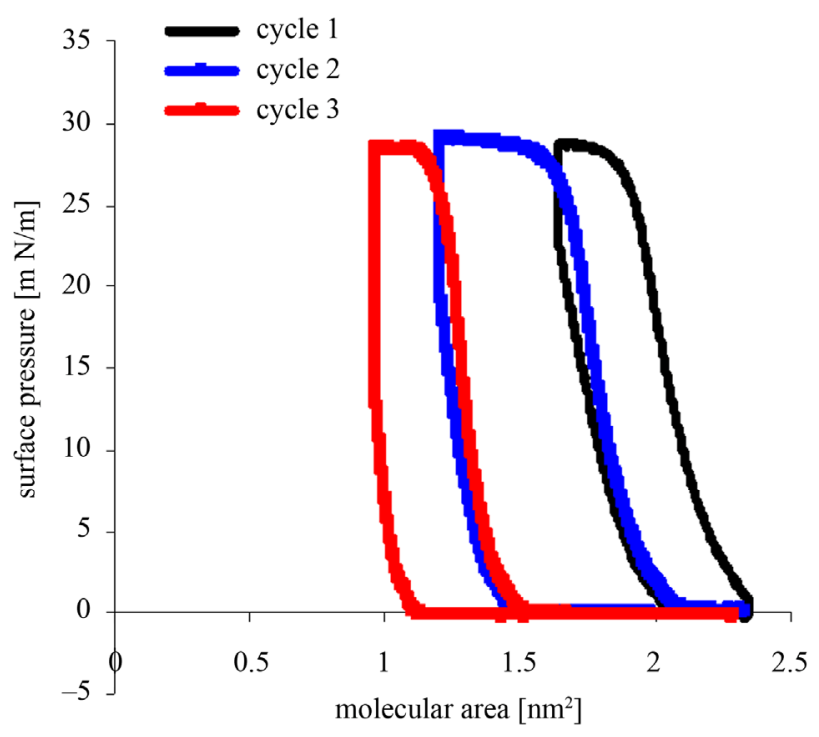

(a)

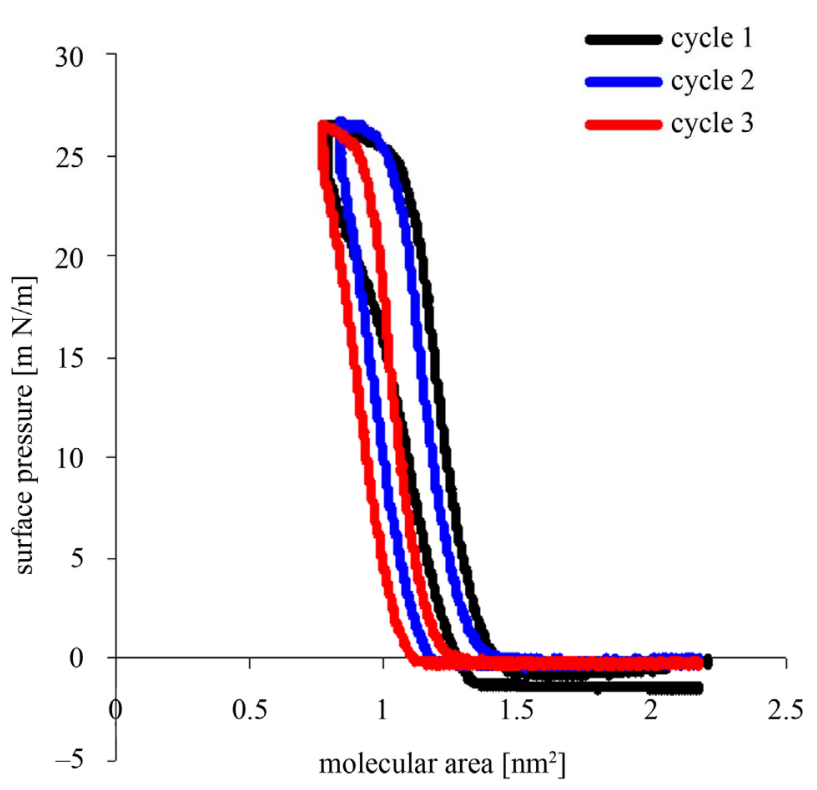

(b)

Figure 2. (a) Representative surface pressure/area isotherms for compression-decompression cycles of Langmuir films GDNT (30 $\mu \mathrm{L}$ of $1 \mathrm{mM}$ ) after $1 \mathrm{~h}$ spreading at the air-water interface; (b) Representative surface pressure/area isotherms for compressiondecompression cycles of Langmuir films GDNT (30 $\mu \mathrm{L}$ of $1 \mathrm{mM}$ ) after $15 \mathrm{~h}$ spreading at the air-water interface.

showed that Langmuir film GDNT area per molecule ranging from $14 \mathrm{~nm}^{2}$ for the first compression-decompression cycle and $11 \mathrm{~nm}^{2}$ at the seventh cycle. Dote et al. [13] and Elferinck et al. [14] reported that GDNT molecules are organised in an upright standing configuration with a molecular area of $0.82 \mathrm{~nm}^{2}$ in the monomolecular films. In monolayer experiments with GDNT from Sulfolobus acidocaldarius, De Rosa [15] found molecular areas between $1.0 \mathrm{~nm}^{2}$ and $1.6 \mathrm{~nm}^{2}$. And many determined from different research groups [16] were found molecular area of GDNT between $0.8 \mathrm{~nm}^{2}-1.0 \mathrm{~nm}^{2}$. From this experiment, we report an interesting phenomenon studied the stability behavior of Langmuir monolayer GDNT tetraether lipid at air-water interface with different spreading time.

The film pressure $\pi$ is defined as the difference between the surface tension of pure solvent $\gamma_{0}$ (generally pure water) and that of $\gamma$-covered surface of the film. Compression state of the film is defined in terms of surface pressure $(\pi)$, defined as:

$$
\pi=\gamma_{\mathrm{o}}-\gamma
$$

where $\pi$ is defined as the surface pressure or differential surface tension, $\gamma_{\mathrm{o}}$ is the surface tension of the gas/liquid interface and $\gamma$ is the surface tension of the gas/film interface. We can best observe whether or not this is in accordance with the usual three-dimensional concept of a pressure through thermodynamic arguments. Amphiphile monolayer formation associated with certain thermodynamic properties of air-water interface. Surface pressure $(\pi)$-area (A) isotherm is a plot of the change in surface pressure as a function of the area available to each molecule on the aqueous subphase surface. Behaviour of the spread of the film can be determined from this curve. This isotherm is the most common indicator of monolayer formation and properties of a monolayer of amphiphilic materials.

We explored the molecular properties of the Langmuir films GDNT by analyzing the surface pressure-area $(\pi-\mathrm{A})$ isotherms of $30 \mu \mathrm{l}$ of $1 \mathrm{mmol} / \mathrm{l} \mathrm{GDNT}$ after 1 hour and 15 hours of spreading on the subphase at the air-water interface. The isotherms exhibited significantly different compression-decompression profiles for different structural features of tetraether lipids GDNT after two different times longer to spread.

Dynamic compression-decompression Langmuir films of GDNT at the air-water interface provides detailed information on molecular dimensions, packing properties and phase transitions of the film. In addition, measuring the compression-decompression cycle provides information about the stability of the film, feature thermodynamics and kinetics.

Thermodynamic features were observed for the compression-decompression of Langmuir monolayer films asymmetrical GDNT tetraether lipid with different spreading times is shown in Figure 2. Langmuir monolayer films stability of GDNT is checked by performing hysteresis experiments, in which the film was compressed to surface pressure-area fixed and then relaxed to its original state. Compression-decompression cycles performed hysteresis and compression-decompression so that the curves show thermodynamic stability of the Langmuir monolayer films of GDNT. Hysteresis curves were observed for the stable 
films $[9,10]$.

Figure 2(a) shows the Langmuir films of $30 \mu 1$ of 1 $\mathrm{mmol} / \mathrm{l}$ of the asymmetric GDNT tetraether lipids 1 hour after spreading at the air-water interface. The first cycle shows that the film is the most stable at a surface pressure of around $28 \mathrm{mN} / \mathrm{m}$ and a molecular area of between $1.8 \mathrm{~nm}^{2}$ and $2.2 \mathrm{~nm}^{2}$. The second cycle also showed that the highest surface pressure was $28 \mathrm{mN} / \mathrm{m}$, but with sharp little smaller molecular area of between $1.3 \mathrm{~nm}^{2}$ and 1.8 $\mathrm{nm}^{2}$. For the third cycle the molecular area was continuous and sharp, between $0.9 \mathrm{~nm}^{2}$ and $1.2 \mathrm{~nm}^{2}$, and the highest surface pressure of only $27 \mathrm{mN} / \mathrm{m}$.

Compression-decompression Langmuir films of $30 \mu 1$ of $1 \mathrm{mmol} / 1$ GDNT tetraether lipids after 15 hours of spreading on the subphase at the air-water interface is shown in Figure 2(b). The first cycle was found to have the highest surface pressure around $25 \mathrm{mN} / \mathrm{m}$ and a molecular area of between $1.4 \mathrm{~nm}^{2}$ and $0.9 \mathrm{~nm}^{2}$. The second cycle also showed the most stable surface pressure of $25 \mathrm{mN} / \mathrm{m}$ and a molecular area that was almost the same as in the first cycle (between $1.4 \mathrm{~nm}^{2}$ and $0.9 \mathrm{~nm}^{2}$ ). In the third cycle the molecular area is slightly smaller, between 1.2 $\mathrm{nm}^{2}$ and $0.9 \mathrm{~nm}^{2}$, with the highest surface pressure of 25 $\mathrm{mN} / \mathrm{m}$.

Langmuir monolayer films stability of $30 \mu \mathrm{l}$ of $1 \mathrm{mmol} / 1$ GDNT at the air-water interface displayed on a curve generated from the surface pressure-area $(\pi-\mathrm{A})$ isotherms of 1 hour and 15 hours after spreading. A hystersis loop performed for each cycle. For 15 hours after spreading, the hysteresis loop cycles are shown toward lower curve of GDNT after 1 hour of spreading. Langmuir film of GDNT after standing for 15 hours is more stable than the Langmuir film after standing for 1 hour. Bakowsky et al. (2008) [11] also showed that TEL-organization at the air-water interface is strongly dependent on the spreading time and the lateral surface pressure. For poorly stable monolayers, continuous changes of the hysteresis loops towards lower mean molecular areas are observed in isotherms, respectively. This can be attributed to a loss of film-forming molecules in the bulk (the dissolution of the monolayer) or molecules to form bi- and multilayer structures (monolayer collapse) [17].

Analysis of the hysteresis loops show the Langmuir monolayer films stability and thermodynamic features. Langmuir monolayer film of GdNT after 15 hours of spreading more stable than one hour after spreading. Floating monolayer stability is an important parameter in obtaining very high-quality Langmuir-Blodgett films. Although, efforts were made to obtain high-quality Langmuir-Blodget films of tetraether lipids [7,9-11,13-16]. Langmuir-Blodget of tetraether lipid films depends on many parameters such as substrate surface properties (hydrophobic, hydrophilic, aminosilanised) [10], temperature, sub-phase, etc.

Repeated compression and decompression cycles can be necessary to analysis achieve reproducible and produces tracks isotherms stable film. Highly stable Langmuir films are important and determine to get very high quality of Langmuir-Blodget monolayer of tetraether lipids.

\section{REFERENCES}

[1] Kaganer, V.M., Mohwald, H. and Dutta, P. (1999) Structure and phase transitions in Langmuir monolayers. Reviews of Modern Physics, 71, 779-819. doi:10.1103/RevModPhys.71.779

[2] Zheng, S., Strzalka, J., Jones, D.H., Opella, S.J. and Blasie, J.K. (2003) Comparative structural studies of vpu peptides in phospholipid monolayers by X-ray scattering. Biophysical Journal, 84, 2393-2415. doi:10.1016/S0006-3495(03)75045-0

[3] Ryley, S., Chyla, A.T. and Peterson, I.R. (2000) An airstable biomimetic Langmuir-Blodgett bilayer. Thin Solid Films, 370, 294-298. doi:10.1016/S0040-6090(99)00876-7

[4] Bilewicz, R., Sawaguchi, T., Chamberlain, R.V. and Majda, M. (1995) Monomolecular Langmuir-Blodgett films at electrodes. Electrochemistry at single molecule "gate sites". Langmuir, 11, 2256-2266.

doi:10.1021/1a00006a065

[5] Nicolae, C.A., Cantin-Riviere, S., El Abed, A. and Peretti, P. (1997) Study of mixed enzyme/pyrrole derivative langmuir-blodgett films for biosensing application. Langmuir, 13, 5507-5510. doi:10.1021/la970117+

[6] Chang, E.L. (1994) Unusual thermal stability of liposomes made from bipolar tetraether lipids. Biochemical and Biophysical Research Communications, 202, 673679. doi:10.1006/bbrc. 1994.1983

[7] Rolandi, R., Schindler, H., De Rosa, M. and Gambacorta, A. (1986) Monolayers of ether lipids from archaebacteria. European Biophysics Journal, 14, 19-27. doi:10.1007/BF00260399

[8] Franz, H., Dante, S., Wappmannsberger, Th., Petry, W., DeRosa, M. and Rustichelli, F. (1998) An X-ray reflectivity study of monolayers and bilayers of archae lipids on a solid substrate. Thin Solid Films, 327-329, 52-55. doi:10.1016/S0040-6090(98)00586-0

[9] Vidawati, S., Sitterberg, J., Bakowsky, U. and Rothe, U. (2010) AFM and ellipsometric studies on LB films of natural asymmetric and symmetric bolaamphiphilic archaebacterial tetraether lipids on silicon wafers. Colloids and Surfaces B: Biointerfaces, 78, 303-309. doi:10.1016/j.colsurfb.2010.03.015

[10] Vidawati, S., Sitterberg, J., Rothe, U. and Bakowsky, U. (2011) Stability of monomolecular films of archaebacterial tetraether lipids on silicon wafers: A comparison of physisorbed and chemisorbed monolayers. Colloids and Surfaces B: Biointerfaces, 87, 209-216. doi:10.1016/j.colsurfb.2011.05.005

[11] Bakowsky, U., Rothe, U., Antonopoulos, E., Martini, T., Henkel, B. and Freisleben, H.J. (2000) Monomolecular organization of the main tetraether lipid from Thermoplasma acidophilum at the water-air interface. Chemistry 
and Physics of Lipids, 105, 31-42. doi:10.1016/S0009-3084(99)00131-0

[12] Bode, M.L., Buddoo, S.R., Minnaar, S.H. and DuPlessis, C.A. (2008) Extraction, isolation and NMR data of the tetraether lipid calditoglycerocaldarchaeol (GDNT) from Sulfolobus metallicus harvested from a bioleaching reactor. Chemistry and Physics of Lipids, 154, 94-104. doi:10.1016/i.chemphyslip.2008.02.005

[13] Dote, J.L., Barger, W.R., Behroozi, F., Chang, E.L., Lo, S.L.C., Montague, E. and Nagumo, M. (1990) Monomolecular film behavior of tetraether lipids from a thermoacidophilic archaebacterium at the air/water interface. Langmuir, 6, 1017-1023. doi:10.1021/la00095a023

[14] Elferinck, M.G.L., De Wit, J.G., Demel, R., Driessen, A.J.M. and Konings, W.N.J. (1992) Functional reconstitution of membrane proteins in monolayer liposomes from bipolar lipids of Sulfolobus acidocaldarius. Journal of Biological Chemistry, 267, 1375-1381.

[15] De Rosa, M. (1996) Archaeal lipids: Structural features and supramolecular organization. Thin Solid Films, 284285, 13-17. doi:10.1016/S0040-6090(96)08832-3

[16] Dante, S., De Rosa, M., Maccioni, B., Morana, A., Nicolini, C., Rustichelli, F., Troitsky, V.I. and Yang, B. (1995) Thermal, stability of bipolar lipid Langmuir Blodgett films by X-Ray diffraction. Molecular Crystals and Liquid Crystals Science and Technology, 262, 191207. doi:10.1080/10587259508033525

[17] Dynarowicz-Latka, P., Dhanabalan, A. and Oliveira, O.N. (2001) Modern physicochemical research on Langmuir monolayers. Advances in Colloid and Interface Science, 91, 221-293. doi:10.1016/S0001-8686(99)00034-2 